\title{
THE CLASSIFICATION PROBLEM FOR FINITE RANK BUTLER GROUPS
}

\author{
SIMON THOMAS
}

\begin{abstract}
In this paper, we shall consider the complexity of the isomorphism and quasi-isomorphism problems for the finite rank Butler groups, as well as the related question of the complexity of the classification problem for representations of finite posets over various fields.
\end{abstract}

\section{INTRODUCTION}

In 1937, Baer [3] introduced the notion of the type of an element in a torsion-free abelian group and showed that this notion provided a complete invariant for the classification problem for torsion-free abelian groups of rank 1. Since then, despite the efforts of such mathematicians as Kurosh [17] and Malcev [18], no satisfactory system of complete invariants has been found for the torsion-free abelian groups of finite rank $n \geq 2$. Consequently, it was natural to ask whether the classification problem for the higher rank groups was genuinely more difficult than that for the rank 1 groups. In 1998, Hjorth [13] proved that this was indeed the case; and soon afterwards, making essential use of the work of Adams-Kechris [1], Hjorth [13] and Zimmer [22], Thomas [21] proved that the complexity of the classification problem for the torsion-free abelian groups of rank $n$ increases strictly with the rank $n$. Of course, abelian group theorists had long before reached the conclusion that the classification problem for the higher rank groups was hopelessly difficult and had shifted their attention from the study of arbitrary finite rank groups to various restricted subclasses, where further progress could be made. In this paper, we shall consider the complexity of the isomorphism and quasi-isomorphism problems for the class of finite rank Butler groups, which has been the main focus of recent research in torsion-free abelian groups of finite rank.

Research partially supported by NSF Grants. 
Definition 1.1. A finite rank torsion-free abelian group $A$ is said to be a Butler group iff $A$ can be expressed as the (not necessarily direct) sum

$$
A=A_{1}+\cdots+A_{s}
$$

of finitely many rank 1 subgroups $A_{1}, \cdots, A_{s}$. (Equivalently, $A$ can be expressed as a sum of finitely many pure rank 1 subgroups.)

Theorem 1.2. The isomorphism and quasi-isomorphism problems for the finite rank Butler groups are both hyperfinite.

This paper is organized as follows. In Section 2, we shall give a brief review of the basic theory of Borel equivalence relations. In particular, we shall define the notion of a hyperfinite equivalence relation and present some examples which arise naturally in the study of finite rank torsion-free abelian groups. The proof of Theorem 1.2 will be given in Section 3. Finally, in Section 4, we shall discuss the problem of classifying the representations $\operatorname{rep}(S, K)$ of a finite poset $S$ over a field $K$.

Acknowledgements: I would like to thank David Arnold and Alexander Kechris for very helpful discussions concerning the material in this paper.

\section{Hyperfinite Equivalence Relations}

In this section, we shall give a brief review of some of the basic notions of the theory of Borel equivalence relations, focussing on the class of hyperfinite equivalence relations. (For more detailed accounts, see Hjorth-Kechris [14] and JacksonKechris-Louveau [15].)

Let $X$ be a standard Borel space; i.e. a Polish space equipped with its associated $\sigma$-algebra of Borel subsets. Then a Borel equivalence relation on $X$ is an equivalence relation $E \subseteq X^{2}$ which is a Borel subset of $X^{2}$. The Borel equivalence relation $E$ is said to be countable iff every $E$-equivalence class is countable. If $E, F$ are Borel equivalence relations on the standard Borel spaces $X, Y$ respectively, then we say that $E$ is Borel reducible to $F$ and write $E \leq_{B} F$ if there exists a Borel map $f: X \rightarrow Y$ such that $x E y$ iff $f(x) F f(y)$. Such a map $f$ is called a Borel reduction from $E$ to $F$. We say that $E$ and $F$ are Borel bireducible and write $E \sim_{B} F$ if both $E \leq_{B} F$ and $F \leq_{B} E$. Finally we write $E<_{B} F$ if both $E \leq_{B} F$ and $F \not{ }_{B} E$. All of the Borel equivalence relations that we shall consider in this paper 
arise from group actions as follows. Let $G$ be a locally compact second countable group. Then a standard Borel $G$-space is a standard Borel space $X$ equipped with a Borel action $(g, x) \mapsto g \cdot x$ of $G$ on $X$. The corresponding $G$-orbit equivalence relation on $X$, which we shall denote by $E_{G}^{X}$, is a Borel equivalence relation. In fact, by Kechris [16], $E_{G}^{X}$ is Borel bireducible with a countable Borel equivalence relation. Conversely, by Feldman-Moore [9], if $E$ is an arbitrary countable Borel equivalence relation on the standard Borel space $X$, then there exists a countable group $G$ and a Borel action of $G$ on $X$ such that $E=E_{G}^{X}$.

Example 2.1. Let $n \geq 1$ and let $\mathbb{Q}^{n}$ be the canonical $n$-dimensional vector space over $\mathbb{Q}$. Then

$$
R\left(\mathbb{Q}^{n}\right)=\left\{A \leqslant \mathbb{Q}^{n} \mid A \text { contains } n \text { linearly independent elements }\right\}
$$

is a standard Borel space. Note that the natural action of $G L_{n}(\mathbb{Q})$ on the vector space $\mathbb{Q}^{n}$ induces a Borel action on the space $R\left(\mathbb{Q}^{n}\right)$ of rank $n$ groups; and that if $A, B \in R\left(\mathbb{Q}^{n}\right)$, then $A, B$ are isomorphic iff there exists an element $\varphi \in G L_{n}(\mathbb{Q})$ such that $\varphi(A)=B$. It follows that the isomorphism relation $\cong_{n}$ on $R\left(\mathbb{Q}^{n}\right)$ is a countable Borel equivalence relation. Combining Hjorth [13] and Thomas [21], we have that

$$
\left(\cong_{1}\right)<_{B}\left(\cong_{2}\right)<_{B} \cdots<_{B}\left(\cong_{n}\right)<_{B} \cdots
$$

In other words, with respect to Borel reducibility, the complexity of the classification problem for the torsion-free abelian groups of rank $n$ increases strictly with the rank $n$.

The least complex Borel equivalence relations are those which are smooth; i.e. those Borel equivalence relations $E$ on a standard Borel space $X$ for which there exists a Borel map $f: X \rightarrow Y$ into a standard Borel space $Y$ such that

$$
x E y \quad \text { iff } \quad f(x)=f(y) .
$$

By Burgess [5], if $E=E_{G}^{X}$ is the orbit equivalence relation of a Borel action of a Polish group $G$, then $E$ is smooth iff there exists a Borel selector for $E$; i.e. a Borel map $s: X \rightarrow X$ which selects a fixed element from each $E$-class. 
Next in complexity come those Borel equivalence relations $E$ which are Borel bireducible with the Vitali equivalence relation $E_{0}$ defined on $2^{\mathbb{N}}$ by

$$
x E_{0} y \quad \text { iff } \quad x(n)=y(n) \text { for all but finitely many } n .
$$

More precisely, by Harrington-Kechris-Louveau [12], if $E$ is a Borel equivalence relation, then $E$ is nonsmooth iff $E_{0} \leq_{B} E$. Furthermore, by Dougherty-JacksonKechris [8], if $E$ is a countable Borel equivalence relation on a standard Borel space $X$, then the following are equivalent:

(1) $E \leq_{B} E_{0}$.

(2) $E$ is hyperfinite; i.e. there exists an increasing sequence

$$
F_{0} \subseteq F_{1} \subseteq \cdots \subseteq F_{n} \subseteq \cdots
$$

of finite Borel equivalence relations on $X$ such that $E=\bigcup_{n \in \mathbb{N}} F_{n}$. (Here an equivalence relation $F$ is said to be finite iff every $F$-equivalence class is finite.)

Example 2.2. Let $\mathbb{P}$ be the set of primes. Recall that if $A$ is a torsion-free abelian group and $0 \neq a \in A$, then the characteristic $\chi(a)$ of $a$ is defined to be the sequence

$$
\left\langle h_{a}(p) \mid p \in \mathbb{P}\right\rangle \in(\mathbb{N} \cup\{\infty\})^{\mathbb{P}},
$$

where $h_{a}(p)$ is the $p$-height of $a$. Two characteristics $\chi_{1}, \chi_{2} \in(\mathbb{N} \cup\{\infty\})^{\mathbb{P}}$ are said to belong to the same type, written $\chi_{1} \equiv \chi_{2}$, iff the following conditions are satisfied:

- $\chi_{1}(p)=\chi_{2}(p)$ for all but finitely many primes $p$; and

- $\chi_{1}(p)=\infty$ iff $\chi_{2}(p)=\infty$ for all primes $p$.

It is easily checked that $\equiv$ is a countable Borel equivalence relation on the standard Borel space $(\mathbb{N} \cup\{\infty\})^{\mathbb{P}}$ and that $\equiv \sim_{B} E_{0}$. If $0 \neq a \in A$, then the type $\tau(a)$ of $a$ is defined to be the corresponding $\equiv$-equivalence class $[\chi(a)]$. If $A \in R(\mathbb{Q})$ is a rank 1 group, then it is easily checked that $\tau(a)=\tau(b)$ for all $0 \neq a, b \in A$. Hence, in this case, we can define the type $\tau(A)$ of $A$ to be $\tau(a)$, where $a$ is any nonzero element of $A$. By Baer [3], if $A, B \in R(\mathbb{Q})$, then $A \cong B$ iff $\tau(A)=\tau(B)$. It follows that the isomorphism problem for the rank 1 groups is hyperfinite.

Example 2.3. Let $n \geq 1$. If $A, B \in R\left(\mathbb{Q}^{n}\right)$, then $A$ and $B$ are said to be quasiequal, written $A \approx B$, iff $A \cap B$ has finite index in both $A$ and $B$. It is easily 
checked that $\approx$ is a countable Borel equivalence relation on $R\left(\mathbb{Q}^{n}\right)$. (For example, see Thomas [21, Lemma 3.2].) By Thomas [21, Theorem 3.8], the quasi-equality relation $\approx$ on $R\left(\mathbb{Q}^{n}\right)$ is hyperfinite. Once again, this result is proved by finding a suitable complete invariant as follows. For each prime $p \in \mathbb{P}$, let $\mathbb{Z}_{p}$ be the ring of $p$-adic integers and let $\mathbb{Z}_{(p)}=\mathbb{Z}_{p} \cap \mathbb{Q}$. Regard $\mathbb{Q}^{n}$ as an additive subgroup of the $n$-dimensional vector space $\mathbb{Q}_{p}^{n}$ over the field $\mathbb{Q}_{p}$ of $p$-adic numbers. For each $A \in R\left(\mathbb{Q}^{n}\right)$, let

$$
A_{p}=\mathbb{Z}_{(p)} \oplus A \leqslant \mathbb{Q}^{n} \quad \text { and } \quad \widehat{A}_{p}=\mathbb{Z}_{p} \otimes A \leqslant \mathbb{Q}_{p}^{n} .
$$

By Fuchs [11, Lemma 93.3], there exists a decomposition

$$
\widehat{A}_{p}=V_{p}(A) \oplus M_{p}(A),
$$

where $V_{p}(A)$ is a $\mathbb{Q}_{p}$-subspace of $\mathbb{Q}_{p}^{n}$ and $M_{p}(A)$ is a free $\mathbb{Z}_{p}$-module. By Thomas [21, Section 4], $A, B \in R\left(\mathbb{Q}^{n}\right)$, then $A$ and $B$ are quasi-equal iff the following conditions are satisfied:

- $A_{p}=B_{p}$ for all but finitely many primes $p$; and

- $V_{p}(A)=V_{p}(B)$ for all primes $p$.

At this point, it should be fairly clear that this data can be encoded within the Vitali equivalence relation $E_{0}$ and hence that the quasi-equality relation $\approx$ is hyperfinite. For more details, see Thomas [21, Section 4].

The collection of hyperfinite countable Borel equivalence relations has (amongst others) the following basic closure properties, all of which will be needed in the proof of Theorem 1.2. (We have chosen $\mathbb{N}^{+}$as the index set because we wish to reserve the symbol $E_{0}$ for the Vitali equivalence relation.)

Theorem 2.4 (Jackson-Kechris-Louveau [15]). Let $E, F$ and $E_{i}$ for $i \in \mathbb{N}^{+}$be countable Borel equivalence relations on the standard Borel spaces $X, Y$ and $X_{i}$ respectively.

(a) If $X=Y, E \subseteq F$ and $F$ is hyperfinite, then $E$ is also hyperfinite.

(b) If $E \leq_{B} F$ and $F$ is hyperfinite, then $E$ is also hyperfinite.

(c) If $E_{i}$ is hyperfinite for each $i \in \mathbb{N}^{+}$, then the relation $\bigsqcup_{i} E_{i}$, defined on $\bigsqcup_{i} X_{i}=\bigcup_{i} X_{i} \times\{i\}$ by

$$
(x, i) \bigsqcup_{i} E_{i}(y, j) \quad \text { iff } \quad i=j \text { and } x E_{i} y,
$$


is also hyperfinite.

(d) If $E_{1}, \cdots, E_{n}$ are hyperfinite, then the relation $E_{1} \times \cdots \times E_{n}$, defined on $X_{1} \times \cdots \times X_{n}$ by $\left(x_{1}, \cdots x_{n}\right) E_{1} \times \cdots \times E_{n}\left(y_{1}, \cdots y_{n}\right) \quad$ iff $\quad x_{i} E_{i} y_{i}$ for all $1 \leq i \leq n$, is also hyperfinite.

(e) If $X=Y, E \subseteq F, E$ is hyperfinite and every $F$-equivalence class contains only finitely many E-equivalence classes, then $F$ is also hyperfinite.

\section{The Proof of Theorem 1.2}

In this section, we shall present the proof of Theorem 1.2. By Theorem 2.4(c), it is enough to show that for each $n \geq 1$, the isomorphism and quasi-isomorphism relations on

$$
B\left(\mathbb{Q}^{n}\right)=\left\{A \in R\left(\mathbb{Q}^{n}\right) \mid A \text { is a Butler group }\right\}
$$

are both hyperfinite. Fix some $n \geq 1$ and let $\cong$ and $\sim$ denote the isomorphism and quasi-isomorphism relations on $B\left(\mathbb{Q}^{n}\right)$. (Recall that if $A, B \in R\left(\mathbb{Q}^{n}\right)$, then $A, B$ are quasi-isomorphic iff there exists $\varphi \in G L_{n}(\mathbb{Q})$ such that $\varphi(A), \varphi(A)$ are quasi-equal.) Then $\cong$ and $\sim$ are countable Borel equivalence relations on $B\left(\mathbb{Q}^{n}\right)$ and clearly $\cong \subseteq \sim$. Hence, by Theorem 2.4(a), it is enough to find a hyperfinite countable Borel equivalence relation $E$ on $B\left(\mathbb{Q}^{n}\right)$ such that $\sim \subseteq E$.

For each $A \in R\left(\mathbb{Q}^{n}\right)$, let $T(A)=\{\tau(a) \mid 0 \neq a \in A\}$ be the typeset of $A$. Clearly if $C, D$ are rank 1 groups, then

$$
C \sim D \quad \text { iff } \quad C \cong D \quad \text { iff } \quad \tau(C)=\tau(D) .
$$

It follows that if $A, B \in R\left(\mathbb{Q}^{n}\right)$ and $A \sim B$, then $T(A)=T(B)$. Hence it is enough to show that the equivalence relation $E$ defined on $B\left(\mathbb{Q}^{n}\right)$ by

$$
A E B \quad \text { iff } \quad T(A)=T(B)
$$

is a hyperfinite countable Borel equivalence relation.

Lemma 3.1 (Butler [6]). For each $A \in B\left(\mathbb{Q}^{n}\right)$, the typeset $T(A)$ is finite.

Proof. By Butler [6, Proposition 3], if $A$ is a finite rank Butler group, then $A$ is a pure subgroup of a completely decomposable torsion-free abelian group $B$ of finite 
rank. It is easily checked that $T(B)$ is finite; and since $A$ is a pure subgroup of $B$, it follows that $T(A) \subseteq T(B)$.

The next result is implicitly contained in Butler [7]. The following proof is based upon the account in Arnold [2, Theorem 3.3.2]. (Recall that the set of types forms a distributive lattice under the partial ordering defined by: $\sigma \leq \tau$ iff there exist characteristics $\psi \in \sigma, \chi \in \tau$ such that $\psi(p) \leq \chi(p)$ for all primes $p \in \mathbb{P}$.)

Lemma 3.2. If $T$ is a finite set of types, then there exist at most countably many groups $A \in B\left(\mathbb{Q}^{n}\right)$ such that $T(A)=T$.

Proof. For each $A \in B\left(\mathbb{Q}^{n}\right)$ and type $\sigma \in T(A)$, the $\sigma$-socle of $A$ is the pure subgroup defined by

$$
A(\sigma)=\{a \in A \mid \tau(a) \geq \sigma\} \cup\{0\}
$$

Let $\mathbb{Q} A(\sigma)$ be the $\mathbb{Q}$-vector subspace of $\mathbb{Q}^{n}$ generated by $A(\sigma)$. Clearly there are only countably many possibilities for $\mathbb{Q} A(\sigma)$. Hence the lemma is an immediate consequence of the following claim, together with the fact that the quasi-equality relation $\approx$ is a countable Borel equivalence relation on $B\left(\mathbb{Q}^{n}\right)$.

Claim 3.3. Suppose that $A, B \in B\left(\mathbb{Q}^{n}\right)$ satisfy the following conditions:

(i) $T(A)=T(B)=T$; and

(ii) $\mathbb{Q} A(\sigma)=\mathbb{Q} B(\sigma)$ for all $\sigma \in T$.

Then $A$ and $B$ are quasi-equal.

To see this, express $A=A_{1}+\cdots+A_{s}$ as a sum of finitely many pure rank 1 subgroups. For each $1 \leq i \leq s$, let $\sigma_{i}=\tau\left(A_{i}\right) \in T$. Since $A_{i} \leqslant \mathbb{Q} A\left(\sigma_{i}\right)=\mathbb{Q} B\left(\sigma_{i}\right)$, there exists an integer $m_{i} \geq 1$ such that $m_{i} A_{i} \leqslant B\left(\sigma_{i}\right)$. Hence if $m=m_{1} \cdots m_{s}$, then $m A \leqslant B$. Similarly, there exists an integer $m^{\prime} \geq 1$ such that $m^{\prime} B \leqslant A$ and so $A, B$ are quasi-equal.

Combining Lemmas 3.1 and 3.2, it follows that $E$ is a countable Borel equivalence relation on $B\left(\mathbb{Q}^{n}\right)$.

Lemma 3.4. E is hyperfinite.

Proof. By Theorem 2.4(c), it is enough to show that $E \uparrow B_{k}\left(\mathbb{Q}^{n}\right)$ is hyperfinite for each $k \geq 1$, where $B_{k}\left(\mathbb{Q}^{n}\right)=\left\{A \in B\left(\mathbb{Q}^{n}\right)|| T(A) \mid=k\right\}$. From now on, fix some 
$k \geq 1$. Let $X=(\mathbb{N} \cup\{\infty\})^{\mathbb{P}}$ be the space of characteristics and for each $\chi \in X$, let $[\chi]$ be the corresponding type. Then there exists a Borel map $f: B_{k}\left(\mathbb{Q}^{n}\right) \rightarrow X^{k}$,

$$
A \mapsto\left(\chi_{1}(A), \cdots, \chi_{k}(A)\right),
$$

such that $T(A)=\left\{\left[\chi_{1}(A)\right], \cdots,\left[\chi_{k}(A)\right]\right\}$ for each $A \in B_{k}\left(\mathbb{Q}^{n}\right)$. Clearly $f$ is a Borel reduction from $E$ to the countable Borel equivalence relation $F$ on $X^{k}$ defined by

$$
\left(\chi_{1}, \cdots, \chi_{k}\right) F\left(\theta_{1}, \cdots, \theta_{k}\right) \quad \text { iff } \quad\left\{\left[\chi_{1}\right], \cdots,\left[\chi_{k}\right]\right\}=\left\{\left[\theta_{1}\right], \cdots,\left[\theta_{k}\right]\right\} .
$$

Hence, by Theorem 2.4(b), it is enough to show that $F$ is hyperfinite. To see this, note that Theorem 2.4(d) implies that the countable Borel equivalence relation $F_{0}$, defined on $X^{k}$ by

$$
\left(\chi_{1}, \cdots, \chi_{k}\right) F_{0}\left(\theta_{1}, \cdots, \theta_{k}\right) \quad \text { iff } \quad\left(\left[\chi_{1}\right], \cdots,\left[\chi_{k}\right]\right)=\left(\left[\theta_{1}\right], \cdots,\left[\theta_{k}\right]\right),
$$

is hyperfinite. Hence, by Theorem 2.4(e), since each $F$-equivalence class is the union of finitely many $F_{0}$-equivalence classes, it follows that $F$ is also hyperfinite.

This completes the proof of Theorem 1.2.

\section{RePRESENTATiONS OF POSETS OVER FIELDS}

Unfortunately, the proof of Theorem 1.2 provides absolutely no new insights into the isomorphism and quasi-isomorphism problems for the finite rank Butler groups. To understand why this is the case, notice that the key step in the proof is Lemma 3.2, which allows us to shift our focus from the quasi-isomorphism relation to the much simpler relation of equality of typesets. As the experts in the field will immediately recognize, the gap between these two equivalence relations is precisely the classification problem for $\mathbb{Q}$-representations of finite posets. (For example, see Arnold [2, Section 3.3].) And since each finite poset has only countably many $\mathbb{Q}-$ representations, the theory of Borel equivalence relations has nothing to say on this classification problem. This is particularly disappointing since, by Nazarova [19], most finite posets have wild representation type; and a number of mathematicians have asked whether the theory of Borel equivalence relations yields a nontrivial hierarchy within the class of wild representation types. In the remainder of this section, in order to partially address this question, we shall consider the complexity of the classification problems for representations of finite posets over local fields 
of characteristic 0 ; i.e. over the real numbers $\mathbb{R}$, the complex numbers $\mathbb{C}$ and the finite extensions of the fields $\mathbb{Q}_{p}$ of $p$-adic numbers.

We shall begin by recalling some basic definitions. Let $F$ be a field and let $S$ be a finite poset. Then $\operatorname{rep}(S, F)$ is the category with objects

$$
U=\left(U_{0}, U_{s} \mid s \in S\right)
$$

where $U_{0}$ is a finite dimensional $F$-vector space and $\left(U_{s} \mid s \in S\right)$ is a collection of subspaces of $U_{0}$ such that

$$
\text { if } s \leq t \text {, then } U_{s} \subseteq U_{t} \text {, }
$$

together with the obvious morphisms. In particular, two representations $U=$ $\left(U_{0}, U_{s} \mid s \in S\right)$ and $U^{\prime}=\left(U_{0}^{\prime}, U_{s}^{\prime} \mid s \in S\right)$ are isomorphic iff there exists a vector space isomorphism $\varphi: U_{0} \rightarrow U_{0}^{\prime}$ such that $\varphi\left(U_{s}\right)=U_{0}^{\prime}$ for all $s \in S$.

Suppose now that $K$ is a local field of characteristic 0 . Then, by restricting our attention to those representations $U=\left(U_{0}, U_{s} \mid s \in S\right)$ such that $U_{0}=K^{n}$ for some $n \geq 1$, we can regard $\operatorname{rep}(S, K)$ as a standard Borel space.

Theorem 4.1. Let $K$ be a local field of characteristic 0 and let $S$ be a finite poset. Then the classification problem for $\operatorname{rep}(S, K)$ is smooth.

Proof. Let $S$ be a finite poset and let $K$ be a local field. In [4, Section 3], BeliskiiSergeichuk present an explicit reduction of the classification problem for $\operatorname{rep}(S, K)$ to the problem of classifying pairs of square matrices up to simultaneous similarity over $K$ (for arbitrary fields $K$, not just for local fields). It is easily checked that the Beliskii-Sergeichuk reduction defines a Borel map between the corresponding standard Borel spaces. Thus it is enough to show that for each $n \geq 1$, the problem of classifying pairs of $n \times n$ matrices up to simultaneous similarity over $K$ is smooth.

To see this, fix some $n \geq 1$ and let $P_{n}$ be the standard Borel space of ordered pairs $(M, N)$ of $n \times n$ matrices. Regard $P_{n}$ as a $K$-variety and consider the algebraic action of $G L_{n}(K)$ on $P_{n}$ defined by

$$
g \cdot(M, N)=\left(g M g^{-1}, g N g^{-1}\right) .
$$

Then clearly $(M, N),\left(M^{\prime}, N^{\prime}\right) \in P_{n}$ are simultaneously similar over $K$ iff these pairs of matrices lie in the same $G L_{n}(K)$-orbit. By Zimmer [22, Theorem 3.1.3], every $G L_{n}(K)$-orbit on $P_{n}$ is locally closed in the Hausdorff topology. Hence, by 
Zimmer [22, Proposition 2.1.12], the orbit equivalence relation of $G L_{n}(K)$ on $P_{n}$ is smooth.

Theorem 4.1 implies that if $K$ is a local field of characteristic 0 and $S$ is a finite poset, then the classification problem for $\operatorname{rep}(S, K)$ admits complete invariants in a suitably chosen standard Borel space. However, the proof gives no indication of how to actually compute such invariants. This problem has been solved in the case when $K=\mathbb{C}$ by Sergeichuk [20], who presented an algorithm for reducing the matrix associated with a $\mathbb{C}$-representation of a finite poset $S$ to a canonical form. (A full system of invariants for pairs of complex matrices up to simultaneous similarity was obtained earlier by Friedland [11], making use of the basic notions of algebraic geometry.)

\section{Concluding Remarks}

If $E, F$ are Borel equivalence relations on the standard Borel spaces $X, Y$ and $E \leq_{B} F$, then this relationship is often interpreted informally as meaning that the $E$-classification problem is less complicated than the $F$-classification problem (or that these problems are equally complicated if $E \sim_{B} F$.) But while this interpretation is intuitively convincing in many cases, such as the classification problems for the torsion-free abelian groups of different finite ranks, it clearly breaks down for the classification problems considered in this paper. For example, let $S$ be any finite poset such that the isomorphism relation $\cong_{S}$ on $\operatorname{rep}(S, \mathbb{C})$ is wild and let $\cong_{1}$ be the isomorphism relation on the space $R(\mathbb{Q})$ of torsion-free abelian groups of rank 1. Then, combining Example 2.2 and Theorem 4.1, it follows that $\cong_{S}<_{B} \cong_{1}$. However, every abelian group theorist would agree that:

- Baer's classification of the rank 1 groups is the prototypical example of a satisfactory classification by invariants; while

- wild classification problems are much too complicated to admit a satisfactory classification by invariants.

Of course, the root of this apparent paradox lies in our use of vague terms such as "complicated" and "satisfactory". In rigorous mathematical terms, the theory of Borel equivalence relations is best understood as a study of the structure of the possible invariants for various classification problems $(X, E)$; or, equivalently, 
a study of the structure of the corresponding quotient spaces $X / E$. And this interpretation of the relationship $\cong_{S}<_{B} \cong_{1}$ is entirely unproblematic:

- The quotient space $\operatorname{rep}(S, \mathbb{C}) / \cong_{S}$ is a standard Borel space; and there exists an algorithm for reducing the matrix associated with each $\mathbb{C}$-representation of $S$ to a canonical form.

- The quotient space $R(\mathbb{Q}) / \cong_{1}$ is not a standard Borel space; and it is impossible to define canonical forms within $R(\mathbb{Q})$.

\section{REFERENCES}

[1] S. R. Adams and A. S. Kechris, Linear algebraic groups and countable Borel equivalence relations, J. Amer. Math. Soc. 13 (2000), 909-943.

[2] D. M. Arnold, Abelian Groups and Representations of Finite Partially Ordered Sets, CMS Books in Mathematics, Springer-Verlag, 2000.

[3] R. Baer, Abelian groups without elements of finite order, Duke Math. Journal 3 (1937), 68-122.

[4] G. R. Belitskii and V. V. Sergeichuk, Complexity of matrix problems, Linear Algebra Appl. 361 (2003), 203-222.

[5] J. P. Burgess, A selection theorem for group actions, Pacific J. Math. 80 (1979), 333-336.

[6] M.C.R. Butler, A class of torsion-free abelian groups of finite rank, Proc. London Math. Soc. (3) 15 (1965), 680-698.

[7] M.C.R. Butler, Torsion-free modules and diagrams of vector spaces, Proc. London Math. Soc. (3) 18 (1968), 635-652.

[8] R. Dougherty, S. Jackson and A. S. Kechris, The structure of hyperfinite Borel equivalence relations, Trans. Amer. Math. Soc. 341 (1994), 193-225.

[9] J. Feldman and C. C. Moore, Ergodic equivalence relations, cohomology and von Neumann algebras, I, Trans. Amer. Math. Soc. 234 (1977), 289-324.

[10] S. Friedland, Simultaneous similarity of matrices, Adv. Math. 50 (1983), 189-265.

[11] L. Fuchs, Infinite Abelian Groups, Pure and Applied Mathematics Vol. 36, Academic Press, 1970.

[12] Harrington, L., Kechris, A. S., Louveau, A., A Glimm-Effros dichotomy for Borel equivalence relations, J. Amer. Math. Soc. 3 (1990), 903-927.

[13] G. Hjorth, Around nonclassifiability for countable torsion-free abelian groups, in Abelian groups and modules (Dublin, 1998), Trends Math., Birkhäuser, Basel, 1999, 269-292.

[14] G. Hjorth and A. S. Kechris, Borel equivalence relations and classification of countable models, Annals of Pure and Applied Logic 82 (1996), 221-272.

[15] S. Jackson, A. S. Kechris, and A. Louveau, Countable Borel equivalence relations, J. Math. Logic 2 (2002), 1-80. 
[16] A. S. Kechris, Countable sections for locally compact group actions, Ergodic Theory and Dynamical Systems 12 (1992), 283-295.

[17] A. G. Kurosh, Primitive torsionsfreie abelsche Gruppen vom endlichen Range, Ann. Math. 38 (1937), 175-203.

[18] A. I. Malcev, Torsion-free abelian groups of finite rank (Russian), Mat. Sbor. 4 (1938), 45-68.

[19] L. A. Nazarova, Partially ordered sets of infinite type, Math. USSR Izvestija 9 (1975), 911938.

[20] V. V. Sergeichuk, Canonical matrices for linear matrix problems, Linear Algebra Appl. 317 (2000), 53-102.

[21] S. Thomas, The classification problem for torsion-free abelian groups of finite rank, J. Amer. Math. Soc. 16 (2003), 233-258.

[22] R. J. Zimmer, Ergodic Theory and Semisimple Groups, Birkhäuser, 1984.

Mathematics Department, Rutgers University, 110 Frelinghuysen Road, Piscataway, NeW Jersey 08854-8019, USA

E-mail address: sthomas@math.rutgers .edu 